\title{
DESIGN CONSIDERATIONS FOR HIGH-CURRENT SUPERCONDUCTING RFQ'S
}

\author{
J. R. Delayen, C. L. Bohn, W. L. Kennedy, L. Sagalovsky \\ Engineering Physics Division, Argonne National Laboratory \\ Argonne, Illinois 60439 USA
}

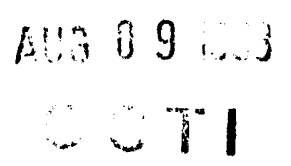

\begin{abstract}
As part of our ongoing development program of highcurrent $\mathrm{cw}$ accelerators, we are investigating the use of superconducting RFQs to capture, bunch, and accelerate high-current, $\mathrm{cw}$ ion beams to energies where they can be further accelerated by more conventional independentlycoupled superconducting cavities. This investigation addresses issues related to beam physics, thermal management, electromagnetic design, mechanical design, matching to the ion source, etc.
\end{abstract}

\section{INTRODUCTION}

As part of our ongoing program to apply rf superconductivity to high-current superconducting accelerators [1], we are investigating the use of superconducting RFQs to capture, bunch, and accelerate high-current ion beams before injection into superconducting linacs comprised of independently-coupled cavities. This research is motivated by preliminary results which indicated that the superconducting technology may appreciably extend the applications of RFQs $[2,3]$. To that end we have been investigating RFQ geometries which seem to be better suited to the superconducting technology [4]. In this paper we address some beam dynamics and design issues associated with the development of high-current superconducting RFQs.

\section{BEA.M DYNa.MICS Issues}

In the beam current range of $25 \mathrm{~mA}$ to $100 \mathrm{~mA}$, thermal management considerations suggest that a tolerable level of beam impingement in a superconducting RFQ (SCRFQ) is $0.5 \%$ or less. Designing a structure with better than $99 \%$ transmissicn presents special challenges. For one, no present beam dynamics code achieves the required accuracy. Another problem is that the design procedures suitable for conventional RFQ's, which use a hard-wired

-Work supported by the Strategic Defense Initiative Organization and the U.S. Department of Energy. prescription based on Kilpatrick factor, field enhancement coefficient, and constant capacitance of the RF structure, may be neither optimal nor desirable for SCRFQ.

As a general guideline, SCRFQ must have a fairly large aperture equal to at least 5 times the rms beam radius. To accommodate the demand for strong transverse focusing, one should choose a low frequency since the superconducting resonators do not seem to suffer from the frequencydependent electric-field breakdown.

Longitudinal transmission is affected by the RFQ bunching rate. The trade-off is between the increased particle capture and the overall length of the accelerator. By increasing the energy at the end of the gentle buncher, thereby causing the beam to be bunched at a slower pace as it is accelerated, we can achieve design transmissions above $99 \%$. The overall length of the RFQ would typically rise quadratically with the energy increase.

As a test case we looked at a SCRFQ design for a $25 \mathrm{~mA}$ and a $100 \mathrm{~mA}$ rms-matched proton beam accelerated from the initial energy of $100 \mathrm{KeV}$ to the final energy of $3 \mathrm{MeV}$. We chose a frequency of $200 \mathrm{M} \mathrm{Hz}$, peak surface field of $40 \mathrm{MV} / \mathrm{m}$, and a minimum aperture of $5 \mathrm{~mm}$ for the beam with initial rms normalized emittance of $0.2 \mathrm{~mm}$ - mrad. We varied the energy at the end of the gentle buncher from $0.5 \mathrm{MeV}$ to $1.5 \mathrm{MeV}$ and studied the transmission through the RFQ with PARMTEQ. The energy at the end of the shaper was kept constant, with exception of the last case where it was raised by $25 \%$. The results are shown in Fig. 1. Fig. 1 also shows how the particle loss relates to the overall $R \cdot Q$ length. For the $25 \mathrm{~mA}$ beam, for example, raising the design beam transmission from $97.7 \%$ to $99.6 \%$ to $99.9 \%$ would mean respective length increases from $2.7 \mathrm{~m}$ to $4.3 \mathrm{~m}$ to $6.0 \mathrm{~m}$. L pon closer examination, one sees that the lower transmission in the shorter structures is due to the particles lost longitudinally. Because of simplifications in the way PARMTEQ calculates spacecharge forces, these "out-of-the-bucket" particles are not accurately tracked throughout the RFQ. It is not clear if the particles are transported unaccelerated or are deflected into the walls of the acclerator. Clearly, the latter scenario would be detrimental to the successful operation of SCRFQ.

We can get a rough understanding of what happens to a 


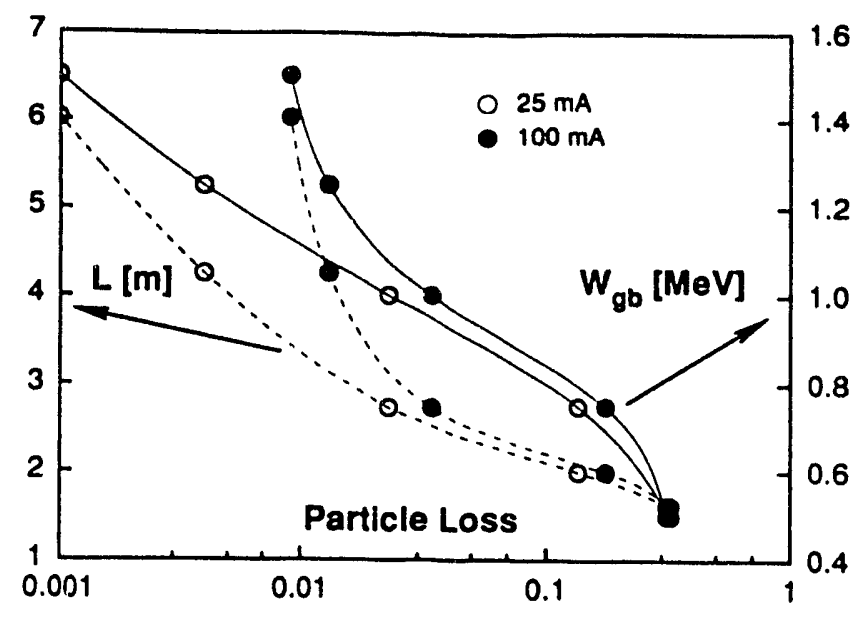

Figure 1: The length $L$ and the energy at the end of gentle buncher $W_{g b}$ as functions of the particle loss. Overall SCRFQ parameters are $f=175 \mathrm{MHz}, E_{9}=40 \mathrm{MV} / \mathrm{m}$, $W_{i}=0.1 \mathrm{MeV}, W_{f}=3.0 \mathrm{MeV}$. Parameters at the end of gentle buncher are $a=0.5 \mathrm{~cm}, m=2.25, \phi_{s}=-30^{\circ}$.

particle which falls out of the longitudinally stable region by calculating the effect of the trailing bunches on its trajectory. The momentum kick imparted on the particle by a bunch of current $I$ can be approximated by a Rutherford scattering formula:

$$
\Delta p_{\perp}=\frac{2 q I}{4 \pi \epsilon_{0} \Delta v f r_{0}}
$$

where $q$ is the particle's charge, $f$ is the RF frequency, $r_{0}$ is the initial radial displacement of the particle, and $\Delta v$ is the relative velocity of the lost particle with respect to the trailing bunch. As the bunch accelerates, its velocity changes but in this rough calculation we assume the velocity to be constant. We take the relative velocity $\Delta v_{n}$ to be

$$
\Delta v_{n}=v_{n}-v_{0}
$$

where $v_{n}$ is the velocity with which the $n^{\text {th }}$ bunch overcomes the lost particle drifting at constant velocity $v_{0}$.

For a particle initially displaced by $r_{0}$ undergoing a betatron oscillation due to the external focusing, we can obtain the oscillation amplitude increase $\Delta r$ due to the spacecharge kick $\Delta p_{\perp}$ :

$$
\Delta r=r_{0}\left[\left(1+\frac{\left(\Delta p_{\perp}\right)^{2}}{m^{2} \Omega^{2} r_{0}^{2}}\right)^{1 / 2}-1\right]
$$

where $m$ is the particle's mass and $\Omega$ is the betatron angular velocity related to the RFQ focusing parameter $B$ and the frequency $f$ as follows,

$$
\Omega \approx \frac{B}{\sqrt{8} \pi} .
$$

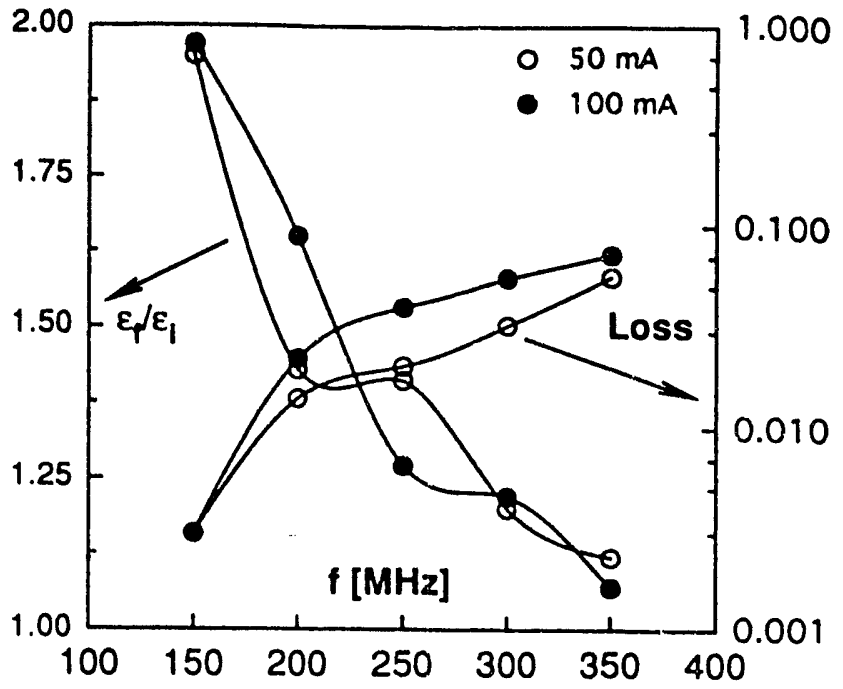

Figure 2: Emittance growth and particle loss versus frequency from PARMTEQ numerical simulation studies of SCRFQ designs for protons at $E,=40 \mathrm{MV} / \mathrm{m}$

For a numerical example, we picked the case mentioned above: a $2.7 \mathrm{mRFQ}$ with the energy at the end of the gentle buncher equal to $1.0 \mathrm{MeV}$. In this design, all the particle loss occurs about $50 \mathrm{~cm}$ upstream the gentle buncher's end; the particle falls out of the bucket when it reaches the energy of $0.32 \mathrm{MeV}$. The design parameters are as follows,

$$
\begin{gathered}
\omega=1.1 \times 10^{7} \mathrm{sec}^{-1} ; \quad f=2.0 \times 10^{8} \mathrm{~Hz} ; \\
\Omega=1.7 \times 10^{8} \mathrm{sec}^{-1} ; \quad r_{0}=0.13 \mathrm{~cm} .
\end{gathered}
$$

Table 1 summarizes the results for the $I=25 \mathrm{~mA}$ design. Table 2 gives the results for the $I=100 \mathrm{~mA}$.

Table 1: Effect of four trailing bunches on a drifting out-of-bucket particle ior $I=25 \mathrm{~mA}$.

\begin{tabular}{|c|c|c|c|c|c|}
\hline$n$ & $w t_{n}$ & $z_{n}[m]$ & $\frac{\Delta v_{n}}{v_{0}}$ & $\frac{\Delta p_{1}}{m v_{0}}$ & $\frac{\Delta_{r_{n}}}{r_{0}}$ \\
\hline 1 & 0.375 & 26.5 & 1.12 & $2.46 \times 10^{-3}$ & $3.8 \times 10^{-3}$ \\
2 & 0.554 & 39.2 & 1.67 & $1.66 \times 10^{-3}$ & $1.7 \times 10^{-3}$ \\
3 & 0.701 & 49.6 & 2.10 & $1.30 \times 10^{-3}$ & $1.0 \times 10^{-3}$ \\
4 & 0.951 & 58.8 & 2.50 & $1.10 \times 10^{-3}$ & $7.5 \times 10^{-4}$ \\
\hline
\end{tabular}

We see that for the $25 \mathrm{mAt}$ beam the radial upward motion of the longitudinally unstable particle is mostly negligible, but for the $100 \mathrm{~mA}$ beam the space-charge imparted radial displacement is on the order of $10 \%$ of the initial radius.

We have also looked at the frequency choice for the SCRFQ. In general, lower frequency is preferable for better transmission characteristics. However, as a rule [j]], lower frequency leads to a larger emittance growth. Fig. 2 shows results of PAR.MTEQ simulations where the particle loss and emittance growth are given as functions of the frequency for $50 \mathrm{m.t}$ and $100 \mathrm{~mA}$ proton beam. 
Table 2: Effect of four trailing bunches on a drifting out-of-bucket particle for $I=100 \mathrm{~mA}$.

\begin{tabular}{|c|c|c|c|c|c|}
\hline$n$ & $w t_{n}$ & $z_{n}[\mathrm{~cm}]$ & $\frac{\Delta v_{n}}{v_{0}}$ & $\frac{\Delta p_{1}}{m v_{0}}$ & $\frac{\Delta r_{n}}{r_{0}}$ \\
\hline 1 & 0.375 & 26.5 & 1.12 & $7.68 \times 10^{-3}$ & $5.9 \times 10^{-2}$ \\
2 & 0.554 & 39.2 & 1.67 & $5.16 \times 10^{-3}$ & $2.7 \times 10^{-2}$ \\
3 & 0.701 & 49.6 & 2.10 & $4.04 \times 10^{-3}$ & $1.7 \times 10^{-2}$ \\
4 & 0.951 & 58.8 & 2.50 & $3.42 \times 10^{-3}$ & $1.2 \times 10^{-2}$ \\
\hline
\end{tabular}

\section{Geometry}

In earlier work [4] we investigated a geometry which is suited to high-current superconducting RFQs. It combined the best features of the 4-vane and 4-rod geometries which are widely used. This geometry is obtained by providing periodic cutouts in a 4-vane geometry which allow magnetic coupling between adjacent quadrants. The resulting structure is simple to manufacture in niobium, is easy to cool, and provides a quadrupole mode which is lower in frequency than, and widely separated from, the dipole mode. We are now extending the analysis of this geometry which was limited to infinite periodic waveguides to include the end effects of a finite RFQ. We have investigated, using MAFIA, the geometry shown in Fig. 3 which consists of $1 / 8$ of an RFQ. The RFQ is terminated by a one-half cell;
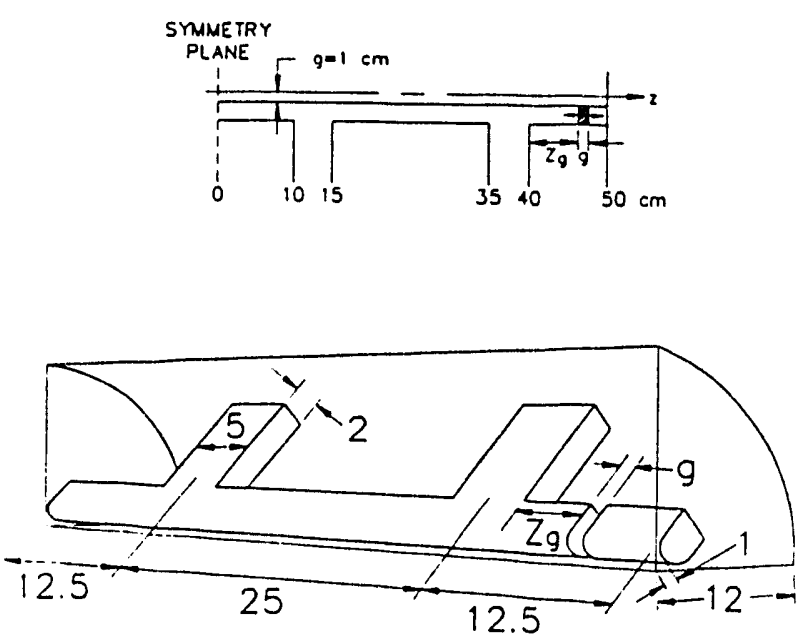

Figure 3: Schematic drawing of $1 / 8$ of the RFQ structure.

the RFQ rods extend a distance $Z_{g}$, and are separated by a distance $g$ (assurned to be of the order of $1 \mathrm{~cm}$ ) from stubs extending from the end plates. Of particular interest is the frequency splitting between the quadrupole and the dipole mode. As shown in Fig. 4, the mode splitting remains almost constant as the length $Z_{g}$ varies from 0 (no extension on the rods) to $10 \mathrm{~cm}$ (no stubs on the end plates). The field flatness, however is affected by the location of the gaps between the rods and the end plate stubs. As shown in Fig. 5, with the end cell dimensions we have assumed, the best field flatness is obtained when the rods

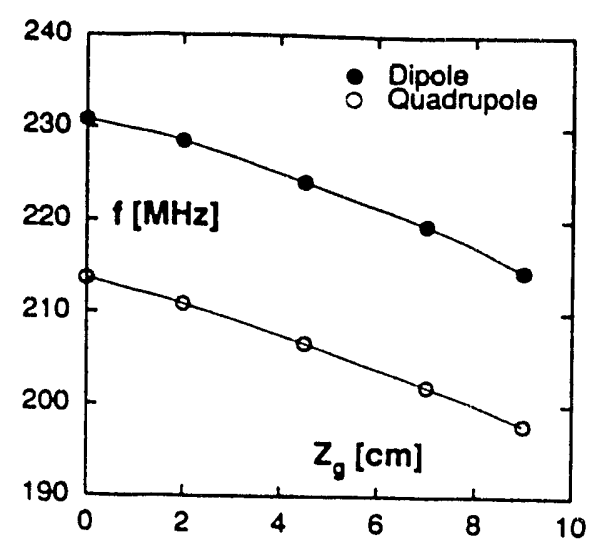

Figure 4: Quadrupole and dipole mode frequencies for structure of Fig. 3 as function of rod extension $Z_{g}$.

extend close to the end plates which have short stubs of less than $1 \mathrm{~cm}$.

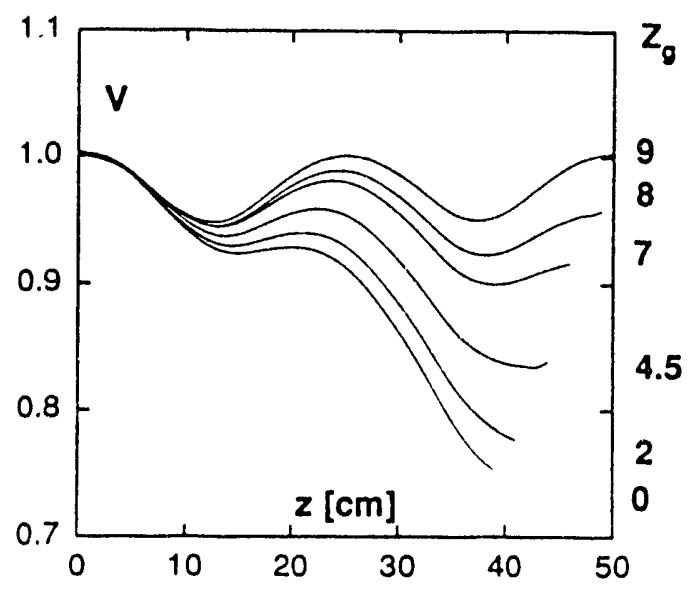

Figure 5: Voltage along the vane (relative units) fo: various rod extensions $Z_{g}$.

\section{REFERENCES}

[1] J.R. Delayen, C.L. Bohn, B.J. Micklich, C.T. Roche, and L. Sagalovsky, "Design Considerations for Highcurrent Superconducting Ion Linacs", these Procs.

[2] J.R. Delayen, K.W. Shepard, Appl. Phys. Letters 57, 514 (1990).

[3] A. Schempp, H. Deitinghoff, J.R. Delayen, K.W. Shepard, Proc. 1990 Linear Accelerator Conference, Los Alamos Report LA-12004-C, 79 (1991).

[4] J.R. Delayen, W.L. Kennedy Proc. 1992 Linear Accelerator Conference AECL-10728 692 (1992).

[j] T.P. Wangler, R.S. Mills, and K.R. Crandall, "Emittance Growth in Intense Beams," Proc. 1987 Particle Accelerstor Conf., IEEE 87CH2387-9, 1006 (1987). 


\section{DISCLAIMER}

This report was prepared as an account of work sponsored by an agency of the United States Government. Neicher the Unired States Governmeen nor any agency thereof. dor any of their employees, mates amy warranty, equress or implied, or arsumes any legal liability or reponsibility for the accoracy, completeneses or use-

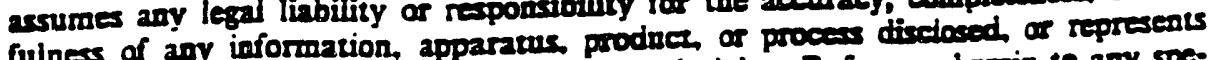
fulness of any information, apparate proded righte Reference berein to ary spethat its use would not iniringe privatedy owned righte Reference berwin to ary spe-

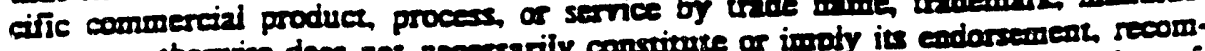

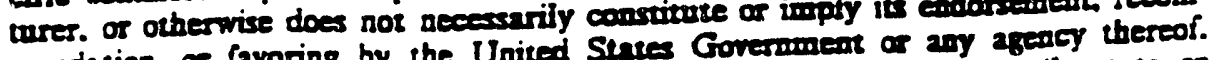

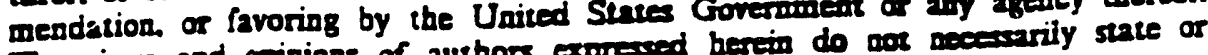
The views and opinions of authors expresed berein do wor mecer.
reflect those of the United States Govermment of any agency thereof. 

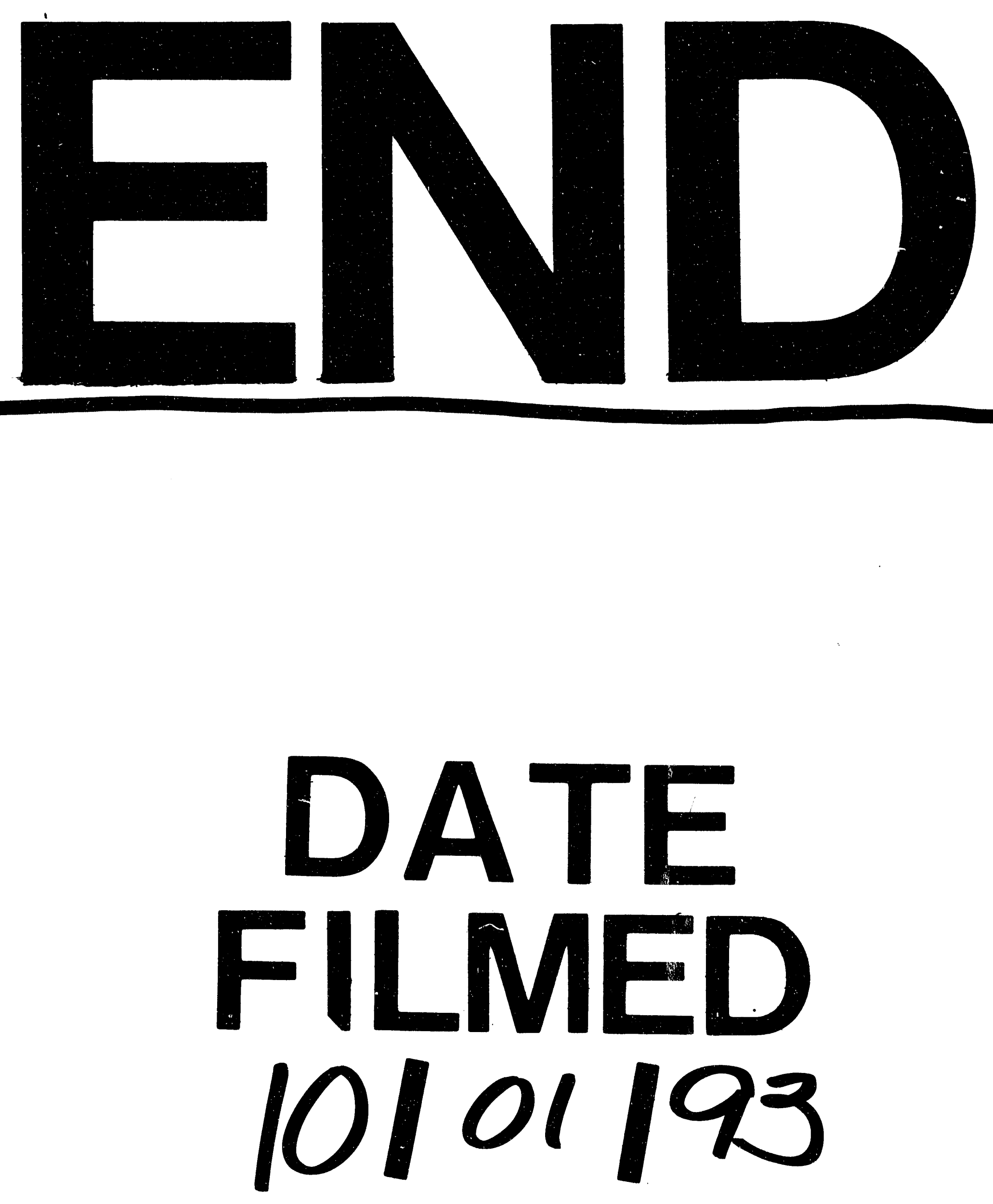
w...

$\Gamma$ 